\title{
Application of Homotopy Perturbation Method for Fuzzy Linear Systems and Comparison with Adomian's Decomposition Method
}

\author{
H. Saberi Najafi, S. A. Edalatpanah, and A. H. Refahi Sheikhani \\ Department of Mathematics, Faculty of Sciences, Islamic Azad University, Lahijan Branch, Lahijan 1616, Iran \\ Correspondence should be addressed to S. A. Edalatpanah; saedalatpanah@gmail.com
}

Received 28 July 2013; Accepted 12 September 2013

Academic Editors: X.-1. Luo, Z. Shi, and Z. Wang

Copyright (C) 2013 H. Saberi Najafi et al. This is an open access article distributed under the Creative Commons Attribution License, which permits unrestricted use, distribution, and reproduction in any medium, provided the original work is properly cited.

We present an efficient numerical algorithm for solution of the fuzzy linear systems (FLS) based on He's homotopy perturbation method (HPM). Moreover, the convergence properties of the proposed method have been analyzed and also comparisons are made between Adomian's decomposition method (ADM) and the proposed method. The results reveal that our method is effective and simple.

\section{Introduction}

Consider the following linear system:

$$
A x=b,
$$

where $x$ denotes a vector in a finite-dimensional space and $A \in R^{n \times n}$. Linear systems are of fundamental importance in various fields of science and engineering and there are numerous methods to find a solution for them; see [1-8] and the references therein.

When coefficients of the system are imprecise, accessing the solution with no ambiguity will be difficult. Therefore, fuzzy logic was proposed by Zadeh (1965) and encountered seriously over Aristotle classic logic. Professor Zadeh discussed fuzzy set theory, fuzzy number concept, and arithmetical operation with these numbers in [9-12] and following that many articles and books were published in fuzzy systems.

We refer the reader to [13] for more information on fuzzy numbers and fuzzy arithmetic. Fuzzy systems are used to study a variety of problems including fuzzy metric spaces [14], fuzzy differential equations [15], particle physics [16, 17], game theory [18], optimization [19], and especially fuzzy linear systems [20-24]. Fuzzy number arithmetic is widely applied and useful in computation of linear system whose parameters are all or partially represented by fuzzy numbers. Friedman et al. [20] introduced a general model for solving a fuzzy $n \times n$ linear system whose coefficient matrix is crisp and the right-hand side column is an arbitrary fuzzy number vector. They used the parametric form of fuzzy numbers and replaced the original fuzzy $n \times n$ linear system by a crisp $2 n \times 2 n$ linear system. Furthermore, some iterative methods have been presented for solving such fuzzy linear systems [25-32].

In this paper, we solve fuzzy linear systems (FLS) via an analytical method called homotopy perturbation method (HPM). This method was first proposed by the Chinese mathematician He in 1999 [33] and was further developed and improved by him [34-36]. This author presented a homotopy perturbation technique based on the introduction of homotopy in topology coupled with the traditional perturbation method for the solution of algebraic equations. This technique provides a summation of an infinite series with easily computable terms, which converges rapidly to the solution of the problem. In the literature, various authors have successfully applied this method for any kinds of different problems [37-42]. The HPM has some advantages over routine numerical methods. This method does not involve discretization of the variables and hence is free from rounding 
off errors and does not require large computer memory or time. In this paper we focus on the use of the homotopy perturbation method for solving the FLS.

\section{Fuzzy Linear Systems (FLS)}

In this section we provide some basic notations and definitions of fuzzy number and fuzzy linear system.

Definition 1. An arbitrary fuzzy number is represented, in parametric form, by an ordered pair of functions $(\underline{u}(r), \bar{u}(r))$, $0 \leq r \leq 1$, which satisfy the following requirements (see [20]):

(i) $\underline{u}(r)$ is a bounded monotonic increasing left continuous function over $[0,1]$;

(ii) $\bar{u}(r)$ is a bounded monotonic decreasing left continuous function over $[0,1]$;

(iii) $\underline{u}(r) \leq \bar{u}(r), 0 \leq r \leq 1$.

A crisp number $\alpha$ can be simply expressed as $\underline{u}(r)=\bar{u}(r)=\alpha$.

The addition and scalar multiplication of fuzzy numbers $x=(\underline{x}(r), \bar{x}(r))$ and $y=(y(r), \bar{y}(r))$ can be described as follows:

(i) $x=y$ if and only if $\underline{x}(r)=\underline{y}(r)$ and $\bar{x}(r)=\bar{y}(r)$,

(ii) $x+y=(\underline{x}(r)+\underline{y}(r), \bar{x}(r)+\bar{y}(r))$,

(iii) $K x=\left\{\begin{array}{l}(K \underline{x}, K \bar{x}), K \geq 0, \\ (K \bar{x}, K \underline{x}), K<0,\end{array} \quad K \in R\right.$.

Definition 2. Consider the following $n \times n$ linear system of equations:

$$
\begin{gathered}
a_{11} x_{1}+a_{12} x_{2}+\cdots+a_{1 n} x_{n}=b_{1}, \\
a_{21} x_{1}+a_{22} x_{2}+\cdots+a_{2 n} x_{n}=b_{2}, \\
\vdots \\
a_{n 1} x_{1}+a_{n 2} x_{2}+\cdots+a_{n n} x_{n}=b_{n},
\end{gathered}
$$

where the coefficient matrix $A=\left(a_{i j}\right), 1 \leq i, j \leq n$, is a crisp matrix and $b_{i} \in E^{1} ; 1 \leq i \leq n$ is called a fuzzy linear system (FLS).

Definition 3. A fuzzy number vector $X=\left(x_{1}, x_{2}, \ldots, x_{n}\right)^{T}$, given by parametric form $x_{i}=\left(x_{i}(r), \overline{x_{i}}(r)\right), 1 \leq i \leq n, 0 \leq$ $r \leq 1$, is called a solution of the fuzzy linear system (2) if

$$
\begin{aligned}
& \underline{\sum_{j=1}^{n} a_{i j} x_{j}}=\sum_{j=1}^{n} \overline{a_{i j} x_{j}}=\underline{b_{i}}, \\
& \overline{\sum_{j-1}^{n} a_{i j} x_{j}}=\sum_{j=1}^{n} \overline{a_{i j} x_{j}}=\overline{b_{i}} .
\end{aligned}
$$

Friedman et al. [20] in order to solve the system given by (3) have solved a $2 n \times 2 n$ crisp linear system as

$$
S X=B,
$$

where $S=\left(s_{i j}\right)$ are determined as follows:

$$
\begin{gathered}
a_{i j} \geq 0 \longrightarrow s_{i j}=a_{i j}, \quad s_{i+n, j+n}=a_{i j}, \\
a_{i j}<0 \longrightarrow s_{i, j+n}=-a_{i j}, \quad s_{i+n, j}=-a_{i j} .
\end{gathered}
$$

And the others are zero. Then refering to [20] we have

$$
\begin{gathered}
S=\left[\begin{array}{ll}
s_{1} & s_{2} \\
s_{2} & s_{1}
\end{array}\right], \quad X=\left[\frac{x}{-\bar{x}}\right], \quad B=\left[\frac{\underline{b}}{-\bar{b}}\right] \\
\Longrightarrow\left\{\begin{array}{c}
s_{1} \underline{x}-s_{2} \bar{x}=\underline{b}, \\
s_{2} \underline{x}-s_{1} \bar{x}=-\bar{b} .
\end{array}\right.
\end{gathered}
$$

Or

$$
\begin{gathered}
S=\left[\begin{array}{cc}
s_{1} & -s_{2} \\
-s_{2} & s_{1}
\end{array}\right], \quad X=\left[\frac{x}{\bar{x}}\right], \quad B=\left[\frac{b}{\bar{b}}\right] \\
\Longrightarrow\left\{\begin{array}{c}
s_{1} \underline{x}-s_{2} \bar{x}=\underline{b}, \\
-s_{2} \underline{x}+s_{1} \bar{x}=\bar{b},
\end{array}\right.
\end{gathered}
$$

where $s_{1}, s_{2} \geq 0$ and $A=s_{1}-s_{2}$.

\section{Homotopy Perturbation Method for Linear Systems}

Consider (1), where

$$
\begin{array}{r}
A=\left[a_{i j}\right], \quad b=\left[b_{i}\right], \quad x=\left[x_{j}\right], \\
i=1,2, \ldots, n, \quad j=1,2, \ldots, n .
\end{array}
$$

Let also $L(u)=A u-b$ and $F(u)=u-w_{0}$, where $w_{0}$ is a known vector. Then we define homotopy $H(u, p)$ as follows:

$$
H(u, p)=(1-p) F(u)+\alpha p L(u)=0,
$$

where $p \in[0,1]$ is an embedding parameter and $\alpha$ is nonzero auxiliary parameter.

Obviously, we will have

$$
\begin{gathered}
H(u, 0)=F(u), \\
H(u, 1)=\alpha L(u)=0 \stackrel{\alpha \neq 0}{\longrightarrow} H(u, 1)=L(u) .
\end{gathered}
$$

According to the HPM, we can first use the embedding parameter $p$ as a small parameter and assume that the solution of (1) can be written as a power series in $p$ :

$$
u=u_{0}+p u_{1}+p^{2} u_{2}+\cdots,
$$

and the exact solution is obtained as follows:

$$
\begin{aligned}
x & =\lim _{p \rightarrow 1} u \\
& =\lim _{p \rightarrow 1}\left(u_{0}+p u_{1}+p^{2} u_{2}+p^{3} u_{3}+\cdots\right) \\
& =\sum_{j=0}^{\infty} u_{j} .
\end{aligned}
$$




$$
\begin{aligned}
& \text { Given a stopping criterion with tolerance } \varepsilon>0 \text {, } \\
& \text { for } n=1,2, \ldots \text {, until } \| \begin{array}{l}
\delta_{n+1}-\delta_{n} \| \leq \varepsilon, \\
\sigma_{n+1}-\sigma_{n} \| \leq \varepsilon,
\end{array} \text { do; } \\
& \delta_{n+1}=\sum_{i=0}^{n}(-1)^{i}\left(\left(\alpha D^{-1} A\right)-I\right)^{i}\left(\alpha D^{-1} b_{1}\right), \\
& \sigma_{n+1}=\sum_{i=0}^{n}(-1)^{i}\left(\alpha\left|D^{-1} A\right|-I\right)^{i}\left(\alpha\left|D^{-1}\right| b_{2}\right), \\
& \text { Obtain } \begin{aligned}
\underline{x} & =\frac{\delta_{n+1}-\sigma_{n+1}}{2}, \\
\bar{x} & =\frac{\delta_{n+1}+\sigma_{n+1}}{2} .
\end{aligned}
\end{aligned}
$$

End for.

Algorithm 1: HPM method for FLS.

Putting (11) into (9) and comparing the coefficients of identical degrees of $p$ on both sides, we find

$$
\begin{aligned}
& p^{0}: u_{0}=w_{0}, \\
& p^{1}:(\alpha A-I) u_{0}+u_{1}-w_{0}-\alpha b=0, \\
& \quad u_{1}=\alpha b-(\alpha A-I) u_{0}+w_{0}, \\
& p^{2}:(\alpha A-I) u_{1}+u_{2}=0, \quad u_{2}=-(\alpha A-I) u_{1}
\end{aligned}
$$

And in general,

$$
u_{n+1}=-(\alpha A-I) u_{n}, \quad n=1,2, \ldots
$$

Taking $u_{0}=w_{0}=0$ yields.

$$
\begin{aligned}
& u_{1}=\alpha b, \\
& u_{2}=-(\alpha A-I) u_{1}=-(\alpha A-I)(\alpha b), \\
& u_{3}=-(\alpha A-I) u_{2}=(\alpha A-I)^{2}(\alpha b) \\
& \vdots \\
& u_{n+1}=-(\alpha A-I) u_{n}=(-1)^{n}(\alpha A-I)^{n}(\alpha b) .
\end{aligned}
$$

Therefore the solution can be in the following form:

$$
x=\sum_{i=0}^{\infty}(-1)^{i}(\alpha A-I)^{i}(\alpha b) .
$$

The convergence of the series equation (16) when $\alpha=1$ is proved for diagonally dominant matrix $A$ in [41].

\section{HPM for the Fuzzy Linear Systems}

Asady and Mansouri in [29] proposed a method for finding a fuzzy solution of $n \times n$ FLS without increasing the order. In this method the original system with a matrix $A$ is replaced by two $n \times n$ crisp linear systems. Furthermore, improvement of computing time compared with other methods by using this method is shown. This method is given by the following.

By noting the structure of $S$ we have the following linear system:

$$
\begin{gathered}
s_{1} \underline{x}+s_{2}(-\bar{x})=\underline{b}, \\
s_{2} \underline{x}+s_{1}(-\bar{x})=-\bar{b} .
\end{gathered}
$$

Therefore, by adding and then subtracting the two parts of (17) we obtain the following:

$$
\begin{aligned}
& A \delta=b_{1}, \\
& E \sigma=b_{2},
\end{aligned}
$$

where

$$
\begin{aligned}
A & =s_{1}-s_{2}, \\
E & =s_{1}+s_{2}, \\
\delta & =(\bar{x}-\underline{x}), \\
\sigma & =(\bar{x}+\underline{x}), \\
b_{1} & =(\bar{b}+\underline{b}), \\
b_{2} & =(\bar{b}-\underline{b}) .
\end{aligned}
$$

Thus, by adding and subtracting the two solutions of systems equation (18) we have the following:

$$
x=\frac{\delta-\sigma}{2}, \quad \bar{x}=\frac{\delta+\sigma}{2} .
$$

Now, we solve the FLS using HPM.

By using the above model, HPM method for FLS is as follows:

$$
\begin{gathered}
\delta=\sum_{i=0}^{\infty}(-1)^{i}\left(\left(\alpha D^{-1} A\right)-I\right)^{i}\left(\alpha D^{-1} b_{1}\right), \\
\sigma=\sum_{i=0}^{\infty}(-1)^{i}\left(\alpha\left|D^{-1} A\right|-I\right)^{i}\left(\alpha\left|D^{-1}\right| b_{2}\right),
\end{gathered}
$$


where $D$ is the diagonal part of $A=D-L-U$ and $-L,-U$ are, respectively, strictly lower and upper triangular matrices of $A$.

So as iterative method we have Algorithm 1.

Next, we will present the convergence analysis of this method.

Definition 4 (see $[1,4,6-8]$ ). (a) A matrix $A=a_{i j}$ is called a $Z$-matrix if for any $i \neq j, a_{i j} \leq 0$.

(b) A $Z$-matrix is a nonsingular $M$-matrix, if $A$ is nonsingular and if $A^{-1} \geq 0$.

(c) For any matrix $A$ the comparison matrix $\langle A\rangle=$ $\left(m_{i j}\right) \in R^{n \times n}$ is defined by

$$
m_{i i}=\left|a_{i i}\right|, \quad m_{i j}=-\left|a_{i j}\right|, \quad i \neq j,
$$

$1 \leq i, j \leq n(|\cdot|$ is symbol of absolute value $)$.

(d) Matrix $A$ is an $H$-matrix if and only if $\langle A\rangle$ is a nonsingular $M$-matrix.

Definition 5 (see $[1,4,6-8]$ ). Let $A$ be a real matrix. The splitting $A=M-N$ is called;

(a) convergent if $\rho\left(M^{-1} N\right)<1$;

(b) $M$-splitting if $M$ is a nonsingular $M$-matrix and $N \geq$ 0 .

Lemma 6 (see [1]). Let $A=M-N$ be an $M$-splitting of $A$. Then $\rho\left(M^{-1} N\right)<1$ if and only if $A$ is a nonsingular $M$-matrix.

Lemma 7 (see [43]). If $A$ is an $H$-matrix, then $\left|A^{-1}\right| \leq\langle A\rangle^{-1}$.

Theorem 8. The series $x=\sum_{i=0}^{\infty}(-1)^{i}\left(\left(\alpha D^{-1} A\right)-I\right)^{i}\left(\alpha D^{-1} b\right)$ in HPM method for solving linear system convergence if $0<$ $\alpha \leq 1$ and $A$ is an H-matrix.

Proof. It is well known that the series convergence if and only if $\rho\left(\alpha D^{-1} A-I\right)<1$.

Furthermore, we have

$$
\begin{aligned}
\rho\left(\alpha D^{-1} A-I\right) & =\rho\left(\alpha D^{-1}\left(A-\alpha^{-1} D\right)\right) \\
& =\rho\left(\alpha D^{-1}\left(\left(\frac{\alpha-1}{\alpha}\right) D-(L+U)\right)\right) .
\end{aligned}
$$

Since $0<\alpha \leq 1$ and $A=D-L-U$ is an $H$-matrix, we see that $D$ is also an $H$-matrix, $\langle A\rangle=\underbrace{(1 / \alpha)|D|}-$ $\underbrace{(((1-\alpha) / \alpha)|D|+|L|+|U|)}_{N}$ is an $M$-matrix, and $\langle A\rangle \stackrel{M}{=} M-N$ is $M$-splitting; by Definition 5 and Lemma 6 we have

$$
\rho\left(\alpha|D|^{-1}\left(\left(\frac{1-\alpha}{\alpha}\right)|D|+(|L|+|U|)\right)\right)<1 .
$$

Furthermore, from Lemma 7,

$$
\begin{gathered}
\left|D^{-1}\right| \leq|D|^{-1} \\
\left|\alpha D^{-1}\left(\frac{1-\alpha}{\alpha} D^{-1}+L+U\right)\right| \\
\leq \alpha\left|D^{-1}\right|\left|\left(\frac{1-\alpha}{\alpha} D^{-1}+L+U\right)\right| \\
\leq \alpha|D|^{-1}\left(\frac{1-\alpha}{\alpha}|D|^{-1}+|L|+|U|\right) .
\end{gathered}
$$

Therefore,

$$
\begin{aligned}
& \rho\left(\alpha D^{-1}\left(\left(\frac{\alpha-1}{\alpha}\right) D-(L+U)\right)\right) \\
& \quad \leq \rho\left(\left|-\alpha D^{-1}\left(\frac{1-\alpha}{\alpha} D+L+U\right)\right|\right) \\
& \quad \leq \rho\left(\alpha|D|^{-1}\left(\left(\frac{1-\alpha}{\alpha}\right)|D|+(|L|+|U|)\right)\right)<1 .
\end{aligned}
$$

And the proof is completed.

Theorem 9. Algorithm 1 for solving the fuzzy linear system converges if $0<\alpha \leq 1$ and $A$ is an H-matrix.

Proof. We need to show that

$$
\max \left(\rho\left(\alpha D^{-1} A-I\right), \quad \rho\left(\alpha\left|D^{-1} A\right|-I\right)\right)<1 .
$$

Also it is easy to see that if $A$ is an $H$-matrix, then $|A|$ is and $H$-matrix too.

Therefore by Theorem 8 the proof is completed.

\section{Comparison Results}

Adomian's decomposition method (ADM) was first introduced by G. Adomian in the beginning of 1980s [44, 45] and has been rapidly growing in recent years. In this method the solution of a functional equation is considered as the sum of an infinite series usually converging to an accurate solution. Allahviranloo in [26] applied the Adomian decomposition method to solve the fuzzy linear systems by model of (6) and has shown that this method is equivalent to the Jacobi iterative method. But since Flop count and the computing time in model (18) are shorter than model (6) we present ADM for FLS based on model (18). This algorithm is as Algorithm 2.

Now, to show the ability of these algorithms we test the following FLS, using Matlab software. 
Given $\begin{aligned} & \delta_{0}=D_{A}^{-1} b_{1}, \\ & \sigma_{0}=D_{E}^{-1} b_{2},\end{aligned}$ and the stopping criterion with tolerance $\varepsilon>0$,

for $n=1,2, \ldots$, until $\begin{aligned} & \left\|\delta_{n+1}-\delta_{n}\right\| \leq \varepsilon, \quad \text {, } \\ & \left\|\sigma_{n+1}-\sigma_{n}\right\| \leq \varepsilon,\end{aligned} \quad$ do

$\delta_{n+1}=D_{A}^{-1}\left(L_{A}+U_{A}\right) \delta_{n}+D_{A}^{-1} b_{1}$,

$\sigma_{n+1}=D_{E}^{-1}\left(L_{E}+U_{E}\right) \sigma_{n}+D_{E}^{-1} b_{2}$,

Obtain $\begin{aligned} \underline{x} & =\frac{\delta_{n+1}-\sigma_{n+1}}{2}, \\ \bar{x} & =\frac{\delta_{n+1}+\sigma_{n+1}}{2} .\end{aligned}$

End for.

ALGORITHM 2: ADM method for FLS.

TABLE 1: Number of iterations of HPM and ADM.

\begin{tabular}{lcc}
\hline$n$ & ITER (HAM) & ITER $($ ADM $)$ \\
\hline 10 & 23 & 32 \\
20 & 41 & 62 \\
30 & 55 & 91 \\
40 & 66 & 119 \\
50 & 80 & 147 \\
60 & 93 & 177 \\
70 & 106 & 204 \\
80 & 119 & 233 \\
90 & 132 & 267 \\
100 & 147 & 290 \\
\hline
\end{tabular}

Example 10. Consider the following $n \times n$ fuzzy system

$$
\begin{aligned}
& 2 x_{1}-x_{3}=(2+r, 4-r), \\
& x_{2}+2 x_{3}-x_{4}=(2+r, 4-r), \\
& x_{3}+2 x_{4}-x_{5}=(2+r, 4-r) \\
& \vdots \\
& x_{n-3}+2 x_{n-2}=(2+r, 4-r), \\
& x_{n-2}+2 x_{n-1}=(2+r, 4-r), \\
& x_{n-1}+2 x_{n}-x_{1}=(2+r, 4-r) .
\end{aligned}
$$

The tables show the numerical results of the above example. In Table 1, we reported the number of iterations (ITER) for the HPM and ADM methods with different $n$. Also $\alpha=0.9$.

From Table 1, we can see that the number of iterations in HPM algorithm is superior to the number of iterations in ADM algorithm.

Tables 2 and 3 also show comparison of accumulated errors for system (28) obtained using HPM and ADM.

From the above tables, we can see that the results of the HPM are close to the exact solution in comparison with ADM which confirm the validity of our method.
TABLE 2: Accumulated error for $\|x\|$ and $n=100$.

\begin{tabular}{lcc}
\hline$r$ & Error $(\mathrm{HAM})$ & Error $(\mathrm{ADM})$ \\
\hline 0.0 & $5.0309 e-005$ & 0.006238873 \\
0.1 & $1.5844 e-004$ & 0.006840745 \\
0.2 & $2.6657 e-004$ & 0.007442617 \\
0.3 & $3.7470 e-004$ & 0.008044489 \\
0.4 & $4.8283 e-004$ & 0.008646361 \\
0.5 & $5.9096 e-004$ & 0.009248233 \\
0.6 & $6.9910 e-004$ & 0.009850104 \\
0.7 & $8.0723 e-004$ & 0.010451976 \\
0.8 & $9.1536 e-004$ & 0.011053848 \\
0.9 & 0.001023488 & 0.011655720 \\
1.0 & 0.001131620 & 0.012257592 \\
\hline
\end{tabular}

TABLE 3: Accumulated error for $\|\bar{x}\|$ and $n=100$.

\begin{tabular}{ccc}
\hline$r$ & Error $(\mathrm{HAM})$ & Error $(\mathrm{ADM})$ \\
\hline 0.0 & $2.7388 e-004$ & 0.006207893 \\
0.1 & $3.6642 e-004$ & 0.006833297 \\
0.2 & $4.5895 e-004$ & 0.007458701 \\
0.3 & $5.5148 e-004$ & 0.008084104 \\
0.4 & $6.4401 e-004$ & 0.008709507 \\
0.5 & $7.3654 e-004$ & 0.009334911 \\
0.6 & $8.2907 e-004$ & 0.009960314 \\
0.7 & $9.2161 e-004$ & 0.010585718 \\
0.8 & 0.001014137 & 0.011211121 \\
0.9 & 0.001106668 & 0.011836525 \\
1.0 & 0.001199200 & 0.012461928 \\
\hline
\end{tabular}

\section{Conclusions}

The homotopy perturbation method (HPM) is a powerful tool which is capable of handling linear/nonlinear equations. In this paper, we proposed homotopy perturbation method and compared it with Adomian's decomposition method (ADM) for solving fuzzy linear systems (FLS). This method does not require parameter in any equation, the same as the perturbation approach, and also is very simple and straightforward. The numerical results show that the results 
of the present method are in excellent agreement with those of exact solution and reductions in the size of calculations compared with the ADM.

\section{Acknowledgments}

The authors would like to thank all the three reviewers for their valuable suggestions which have led to an improvement in both the quality and clarity of this paper.

\section{References}

[1] R. S. Varga, Matrix Iterative Analysis, Springer, Berlin, Germany, 2nd edition, 2000.

[2] D. M. Young, Iterative Solution of Large Linear Systems, Academic Press, New York, NY, USA, 1971.

[3] Y. Saad, Iterative Methods for Sparse Linear Systems, SIAM, Philadelphia, Pa, USA, 2003.

[4] H. S. Najafi and S. A. Edalatpanah, "On the convergence regions of generalized AOR methods for linear complementarity problems," Journal of Optimization Theory and Applications, vol. 156, pp. 859-866, 2013.

[5] H. S. Najafi and S. A. Edalatpanah, "A new modified SSOR ieration method for solving augmented linear systems," International Journal of Computer Mathematics, 2013.

[6] H. S. Najafi and S. A. Edalatpanah, "Comparison analysis for improving preconditioned SOR-type iterative method," Numerical Analysis and Applications, vol. 6, pp. 62-70, 2013.

[7] H. S. Najafi and S. A. Edalatpanah, "Iterative methods with analytical preconditioning technique to linear complementarity problems: application to obstacle problems," RAIRO, vol. 47, pp. 59-71, 2013.

[8] H. S. Najafi and S. A. Edalatpanad, "On application of Liao's method for system of linear equations," Ain Shams Engineering Journal, vol. 4, pp. 501-505, 2013.

[9] L. A. Zadeh, "Fuzzy sets," Information and Control, vol. 8, no. 3, pp. 338-353, 1965.

[10] L. A. Zadeh, "A fuzzy-set-theoretic interpretation of linguistic hedges," Journal of Cybernetics, vol. 2, no. 3, pp. 4-34, 1972.

[11] L. A. Zadeh, "The concept of a linguistic variable and its application to approximate reasoning-I," Information Sciences, vol. 8, no. 3, pp. 199-249, 1975.

[12] L. A. Zadeh, "Fuzzy sets as a basis for a theory of possibility," Fuzzy Sets and Systems, vol. 1, no. 1, pp. 3-28, 1978.

[13] A. Kaufmann and M. M. Gupta, Introduction Fuzzy Arithmetic, Van Nostrand Reinhold, New York, NY, USA, 1985.

[14] J. H. Park, "Intuitionistic fuzzy metric spaces," Chaos, Solitons and Fractals, vol. 22, no. 5, pp. 1039-1046, 2004.

[15] M. Friedman, M. Ming, and A. Kandel, "Numerical solutions of fuzzy differential and integral equations," Fuzzy Sets and Systems, vol. 106, no. 1, pp. 35-48, 1999.

[16] M. S. Elnaschie, "A review of E infinity theory and the mass spectrum of high energy particle physics," Chaos, Solitons and Fractals, vol. 19, no. 1, pp. 209-236, 2004.

[17] M. S. Elnaschie, "The concepts of E infinity: an elementary introduction to the Cantorian-fractal theory of quantum physics," Chaos, Solitons and Fractals, vol. 22, no. 2, pp. 495-511, 2004.

[18] H. S. Najafi and S. A. Edalatpanad, "On the Nash equilibrium solution of fuzzy bimatrix games," International Journal of Fuzzy Systems and Rough Systems, vol. 5, no. 2, pp. 93-97, 2012.
[19] H. S. Najafi and S. A. Edalatpanad, "A note on 'a new method for solving fully fuzzy linear programming problems"' Applied Mathematical Modelling, vol. 37, pp. 7865-7867, 2013.

[20] M. Friedman, M. Ming, and A. Kandel, "Fuzzy linear systems," Fuzzy Sets and Systems, vol. 96, no. 2, pp. 201-209, 1998.

[21] D. Dubois and H. Prade, Fuzzy Sets and Systems: Theory and Applications, Academic Press, NewYork, NY, USA, 1980.

[22] D. Dubois and H. Prade, "Systems of linear fuzzy constraints," Fuzzy Sets and Systems, vol. 3, no. 1, pp. 37-48, 1980.

[23] J. J. Buckley and Y. Qu, "Solving systems of linear fuzzy equations," Fuzzy Sets and Systems, vol. 43, no. 1, pp. 33-43, 1991.

[24] M. Friedman, M. Ming, and A. Kandel, "Duality in fuzzy linear systems," Fuzzy Sets and Systems, vol. 109, pp. 55-58, 2000.

[25] T. Allahviranloo, "Numerical methods for fuzzy system of linear equations," Applied Mathematics and Computation, vol. 155, no. 2, pp. 493-502, 2004.

[26] T. Allahviranloo, "The Adomian decomposition method for fuzzy system of linear equations," Applied Mathematics and Computation, vol. 163, no. 2, pp. 553-563, 2005.

[27] M. Dehghan and B. Hashemi, "Iterative solution of fuzzy linear systems," Applied Mathematics and Computation, vol. 175, no. 1, pp. 645-674, 2006.

[28] K. Wang and B. Zheng, "Symmetric successive overrelaxation methods for fuzzy linear systems," Applied Mathematics and Computation, vol. 175, no. 2, pp. 891-901, 2006.

[29] B. Asady and P. Mansouri, "Numerical solution of fuzzy linear system," International Journal of Computer Mathematics, vol. 86, no. 1, pp. 151-162, 2009.

[30] H. S. Najafi and S. A. Edalatpanah, "The block AOR iterative methods for solving fuzzy linear systems," The Journal of Mathematics and Computer Science, vol. 4, pp. 527-535, 2012.

[31] H. S. Najafi and S. A. Edalatpanah, "Preconditioning strategy to solve fuzzy linear systems (FLS)," International Review of Fuzzy Mathematics, vol. 7, no. 2, pp. 65-80, 2012.

[32] H. S. Najafi and S. A. Edalatpanad, "An improved model for iterative algorithms in fuzzy linear systems," Computational Mathematics and Modeling, vol. 24, pp. 443-451, 2013.

[33] J. He, "Homotopy perturbation technique," Computer Methods in Applied Mechanics and Engineering, vol. 178, no. 3-4, pp. 257262, 1999.

[34] J. He, "Homotopy perturbation method: a new nonlinear analytical technique," Applied Mathematics and Computation, vol. 135, no. 1, pp. 73-79, 2003.

[35] J. He, "Application of homotopy perturbation method to nonlinear wave equations," Chaos, Solitons and Fractals, vol. 26, no. 3, pp. 695-700, 2005.

[36] J. He, "Homotopy perturbation method for solving boundary value problems," Physics Letters A, vol. 350, no. 1-2, pp. 87-88, 2006.

[37] A. Beléndez, A. Hernández, T. Beléndez, E. Fernández, M. L. Alvarez, and C. Neipp, "Application of He's homotopy perturbation method to the Duffing-harmonic oscillator," The International Journal of Nonlinear Sciences and Numerical Simulation, vol. 8, pp. 79-88, 2007.

[38] T. Öziş and A. Yildirim, "A note on He's homotopy perturbation method for van der Pol oscillator with very strong nonlinearity," Chaos, Solitons and Fractals, vol. 37, no. 3, pp. 989-991, 2007.

[39] J. I. Ramos, "Series approach to the Lane-Emden equation and comparison with the homotopy perturbation method," Chaos, Solitons and Fractals, vol. 38, no. 2, pp. 400-408, 2008. 
[40] L. Cveticanin, "Application of homotopy-perturbation to nonlinear partial differential equations," Chaos, Solitons and Fractals, vol. 40, no. 1, pp. 221-228, 2009.

[41] B. Keramati, "An approach to the solution of linear system of equations by He's homotopy perturbation method," Chaos, Solitons and Fractals, vol. 41, no. 1, pp. 152-156, 2009.

[42] M. Mehrabinezhad and J. Saberi-Nadjafi, "Application of He's homotopy perturbation method to linear programming problems," International Journal of Computer Mathematics, vol. 88, no. 2, pp. 341-347, 2011.

[43] A. Frommer and D. B. Szyld, "H-splittings and two-stage iterative methods," Numerische Mathematik, vol. 63, no. 1, pp. 345-356, 1992.

[44] G. Adomian, Solving Frontier Problems of Physics: The Decomposition Method, Kluwer Academic, Dordrecht, The Netherlands, 1994.

[45] G. Adomian and R. Rach, "On the solution of algebraic equations by the decomposition method," Journal of Mathematical Analysis and Applications, vol. 105, no. 1, pp. 141-166, 1985. 


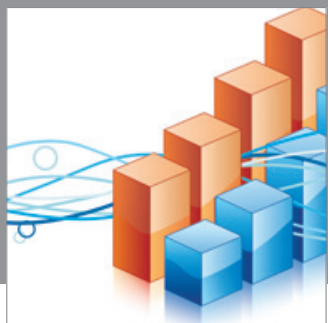

Advances in

Operations Research

mansans

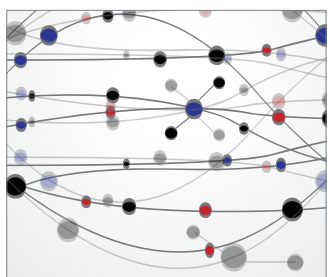

The Scientific World Journal
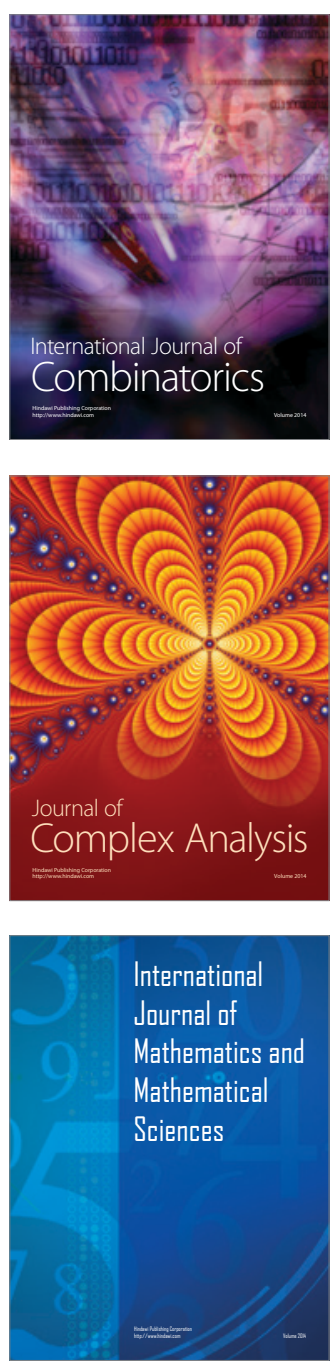
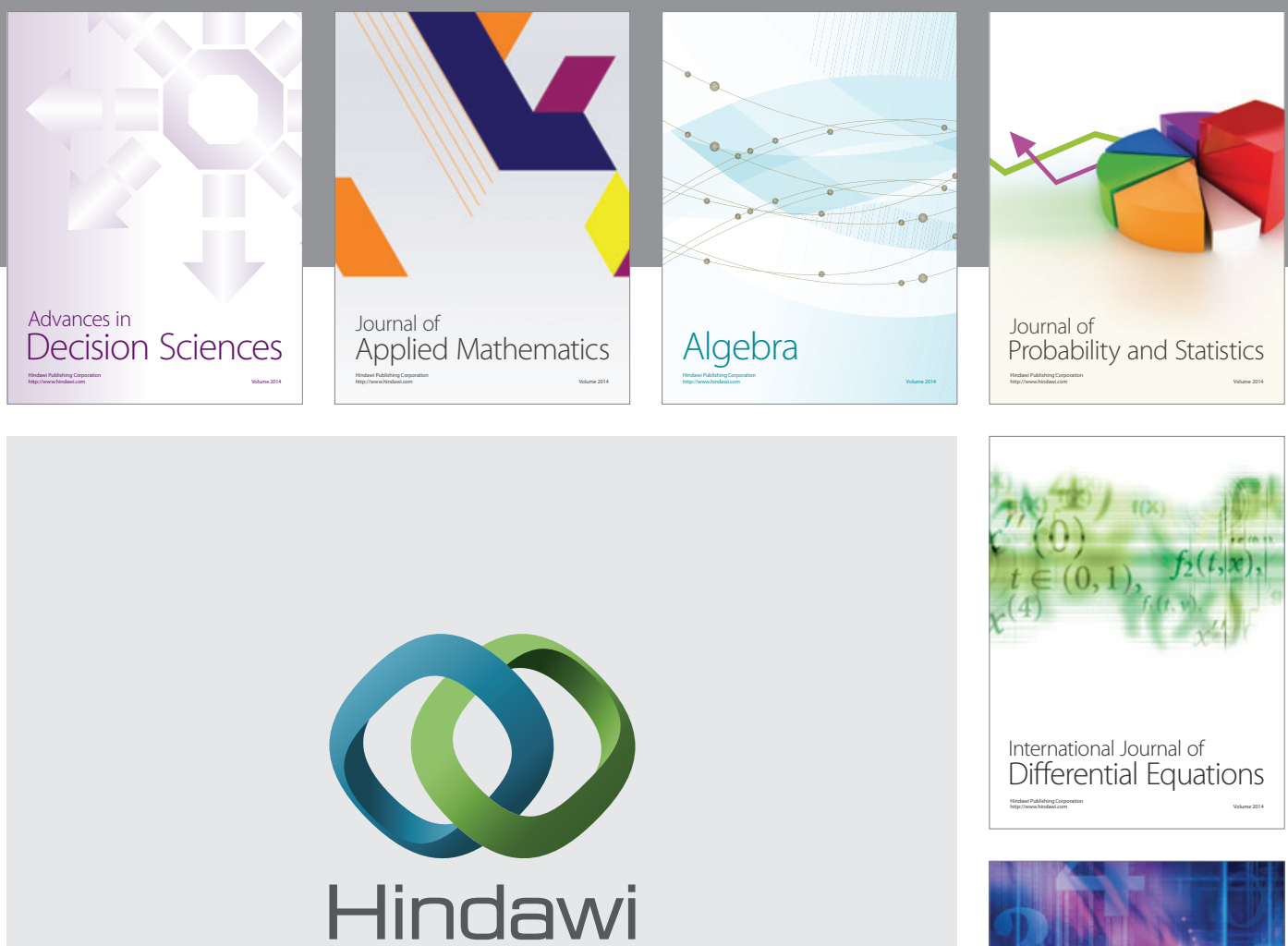

Submit your manuscripts at http://www.hindawi.com
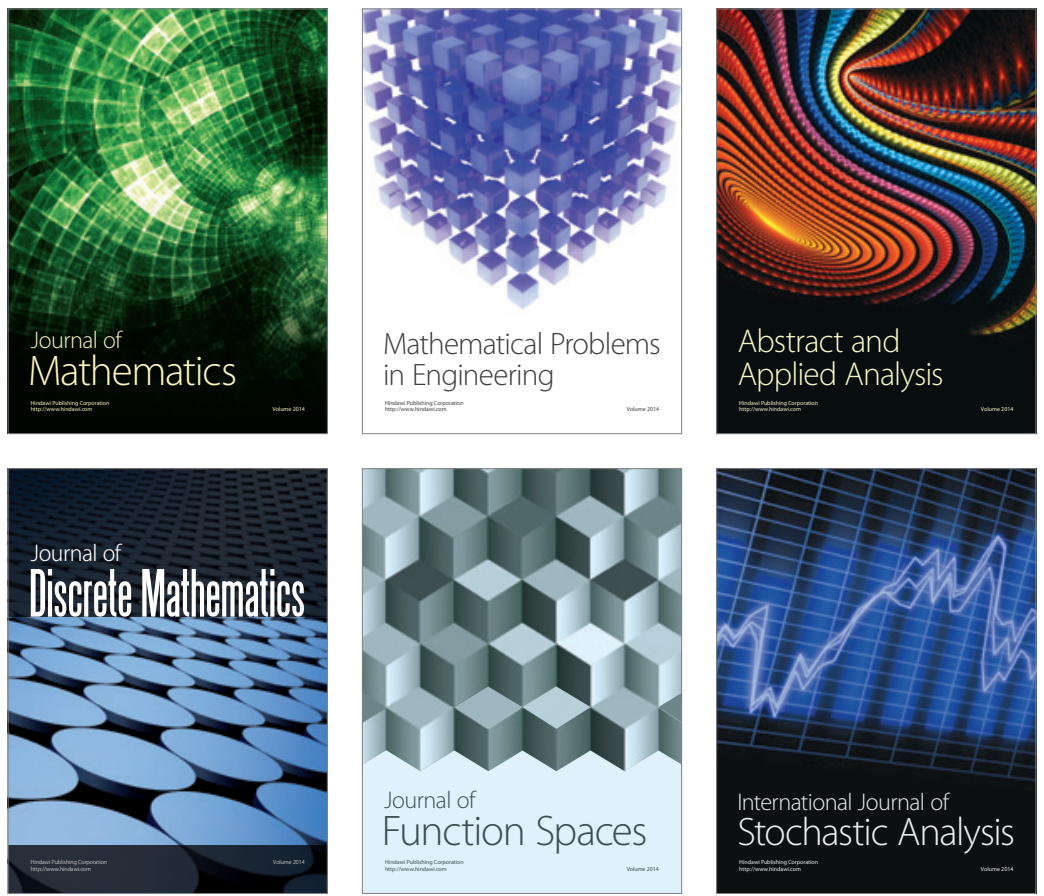

Journal of

Function Spaces

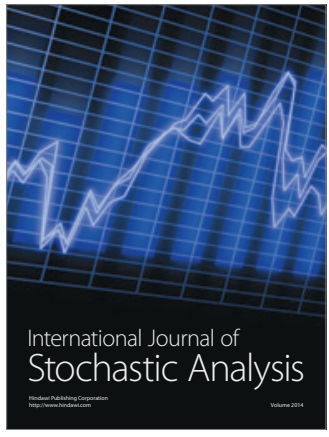

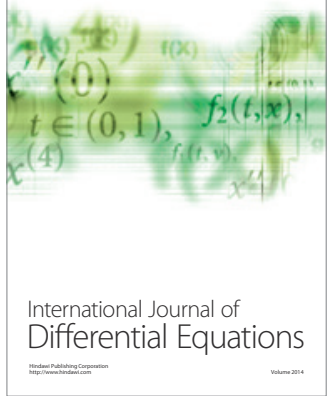
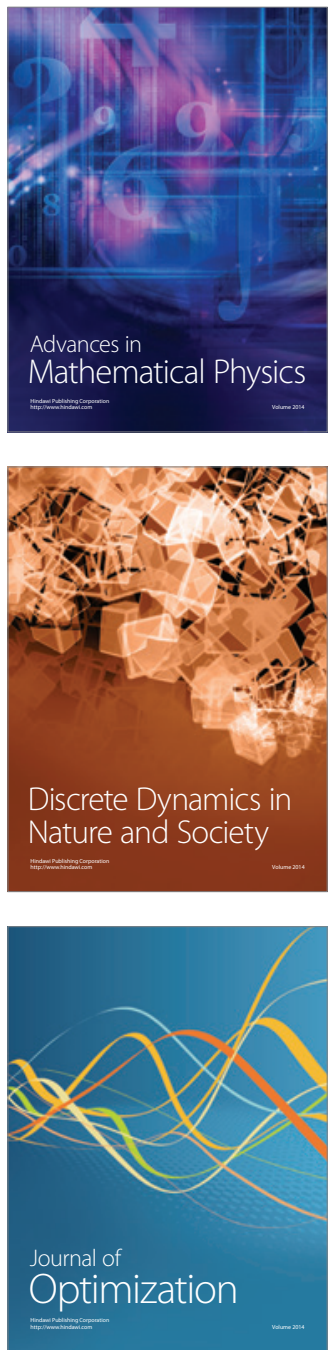